\title{
Naked-Eye Detection of Hydrogen Peroxide on Photoluminescent Paper Discs
}

\author{
Arnab Dutta and Uday Maitra*
}

Department of Organic Chemistry, Indian Institute of Science, Bengaluru, Karnataka 560012, India

$$
\text { E-mail: *maitra@iisc.ac.in }
$$

\section{Synthesis of 2':}

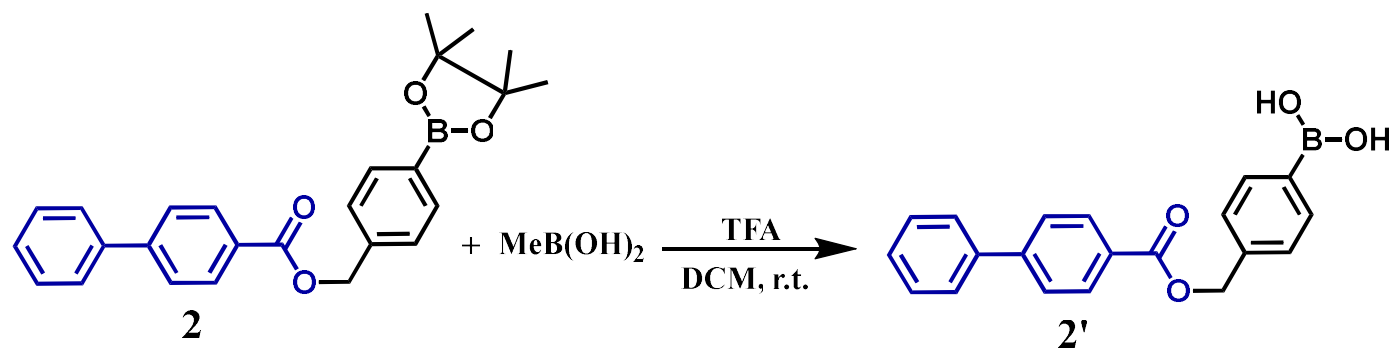

To a mixture of 2 (16 mg, $0.04 \mathrm{mmol}, 1 \mathrm{eq})$ and methylboronic acid ( $8 \mathrm{mg}, 0.13 \mathrm{mmol}, 3.2 \mathrm{eq})$ taken in a round-bottomed flask $(5 \mathrm{~mL})$, equipped with a magnetic stir bar, $5 \%$ TFA solution in dry DCM $(0.7 \mathrm{~mL})$ was added. The reaction was continued overnight under a nitrogen atmosphere with constant stirring. The reaction mixture was concentrated on a rotary evaporator and dried under vacuum to get an off-white solid as the product (12 $\mathrm{mg}, 93 \%)$.

${ }^{1}$ H NMR (400 MHz, DMSO-D 6 with added $\left.D_{2} \mathrm{O}\right) \delta$ ppm: 8.07-8.05 (d, j= 8.4 Hz, 2H), 7.83-7.71 (m, $6 \mathrm{H})$, 7.51-7.41 (m, 5H), $5.36(\mathrm{~s}, 2 \mathrm{H})$. (Figure S12)

${ }^{13}$ C NMR (100 MHz, DMSO-D 6 with added $\left.\mathbf{D}_{2} \mathrm{O}\right) \boldsymbol{\delta}$ ppm: 165.99, 145.33, 139.16, 138.40, 134.66, $130.37,29.57,128.91,128.73,127.43,127.37,127.26,66.56$. (Figure S13)

HRMS: Calculated for $\mathrm{C}_{20} \mathrm{H}_{17} \mathrm{BO}_{4} \mathrm{Na}[\mathrm{M}+\mathrm{Na}]$ 355.1118, Observed $\mathrm{C}_{20} \mathrm{H}_{17} \mathrm{BO}_{4} \mathrm{Na}[\mathrm{M}+\mathrm{Na}] 355.1124$. 
2. Images of TbCh/EuCh hydrogels gels:
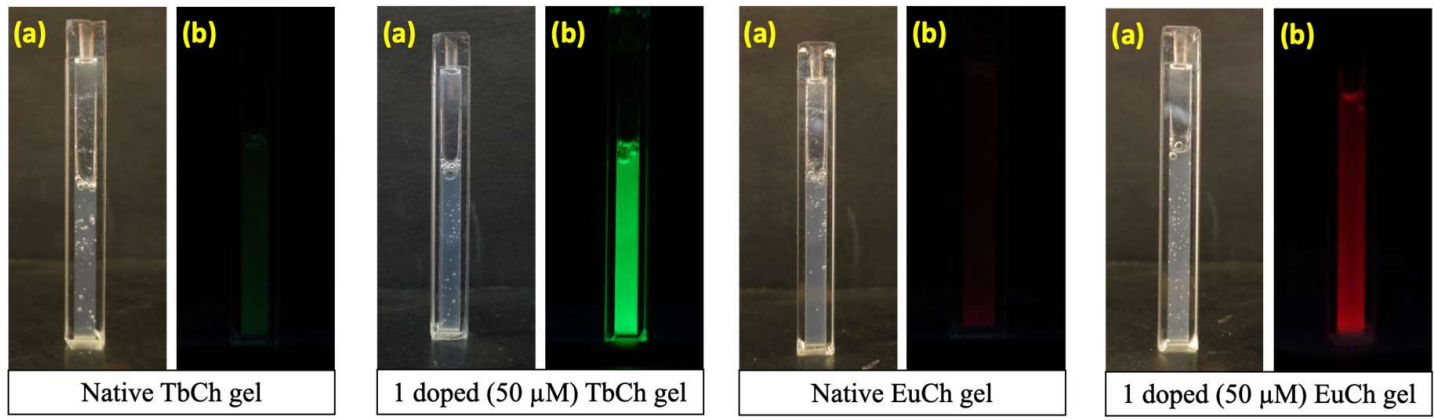

Figure S1: Images of $\mathrm{TbCh} / \mathrm{EuCh}$ hydrogels (a) in ambient light and (b) under UV lamp.

\section{Absorption spectra of 1 and 2:}

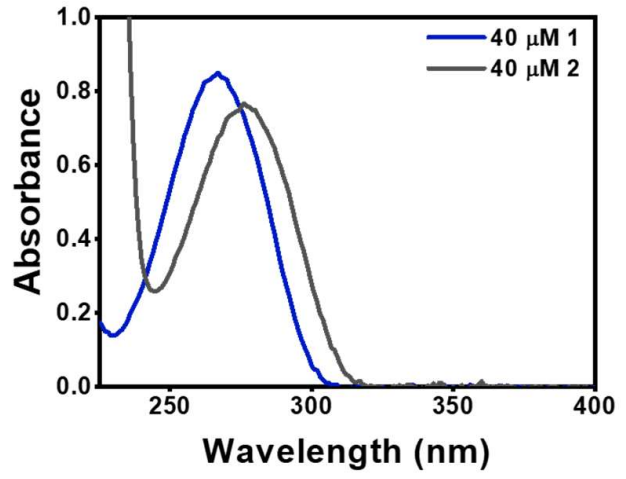

Figure S2: Absorption spectra of $\mathbf{1}$ and $\mathbf{2}$ in $30 \mathrm{mM}$ sodium cholate solution. 
4. Excitation and emission spectra of 1 and 2 doped TbCh gels:

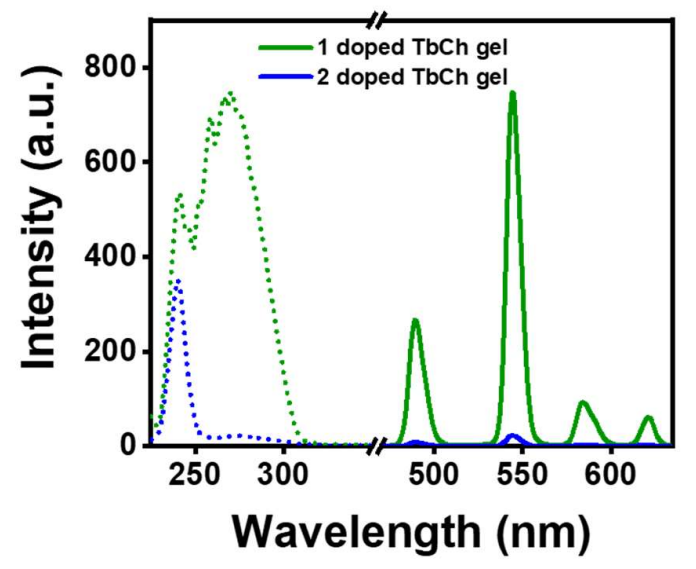

Figure S3: Time-delayed excitation (dotted line: $\lambda_{\mathrm{em}}=545 \mathrm{~nm}$ ) and emission (solid line: $\lambda_{\mathrm{ex}}=270 \mathrm{~nm}$ ) spectra of $1(50 \mu \mathrm{M})$ and $\mathbf{2}(50 \mu \mathrm{M})$ doped TbCh gels.

5. Luminescence decay profile of $1 / 2$ doped and native TbCh gels:

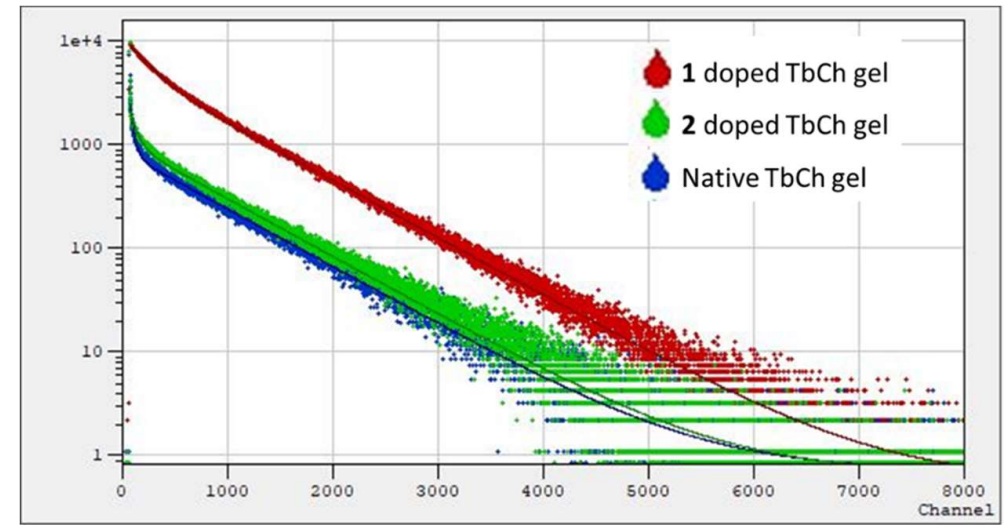

Figure S4: Luminescence decay profile of $1(50 \mu \mathrm{M}), 2(50 \mu \mathrm{M})$ doped and native TbCh gels $\left(\lambda_{\mathrm{em}}=545\right.$ $\left.\mathrm{nm}, \lambda_{\mathrm{ex}}=270 \mathrm{~nm}\right)$.

6. Table S1: Lifetime data of $\mathbf{T b}^{3+}$.

\begin{tabular}{|c|c|c|}
\hline Gel & $\begin{array}{c}\text { Lifetime }(\mathbf{m s}) \\
\text { Average lifetime (ms) }\end{array}$ \\
\hline 1 doped TbCh gel & $0.52(16.2 \%)+2.0(83.8 \%) ; \chi^{2}=1.10$ & 1.93 \\
\hline $\mathbf{2}$ doped TbCh gel & $0.18(9.0 \%)+1.9(91 \%) ; \chi^{2}=1.30$ & 1.88 \\
\hline Native TbCh gel & $0.09(9.4 \%)+1.9(90.6 \%) ; \chi^{2}=1.28$ & 1.89 \\
\hline
\end{tabular}


7. Absorption spectrum of 2', and excitation \& emission spectra of 1/2' doped TbCh gel:
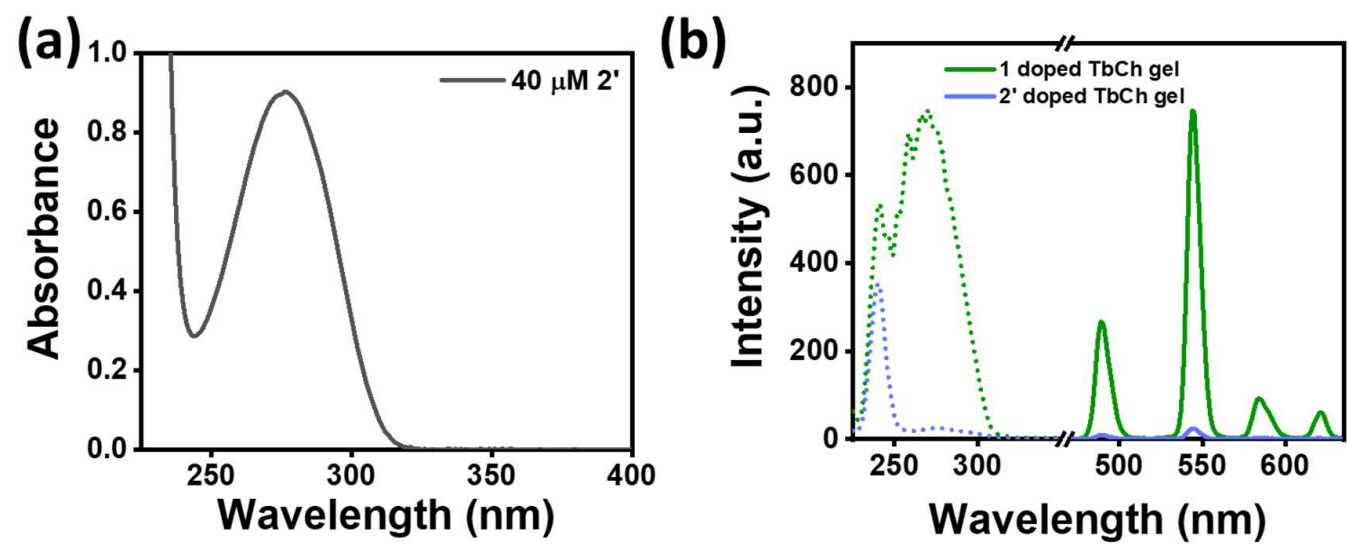

Figure S5: (a) Absorbance spectrum of 2' $(40 \mu \mathrm{M})$ in $30 \mathrm{mM}$ sodium cholate solution. (b) Time-delayed excitation (dotted line: $\lambda_{\mathrm{em}}=545 \mathrm{~nm}$ ) and emission (solid line: $\left.\lambda_{\mathrm{ex}}=270 \mathrm{~nm}\right)$ spectra of $1(50 \mu \mathrm{M})$ and 2' $(50 \mu \mathrm{M})$ doped TbCh gels.

\section{Calculation of LOD (using data recorded in plate reader instrument):}

LOD was calculated using the following equation,

$\mathrm{LOD}=3 \sigma / b$

Where $\sigma$ is the standard deviation for the time-delayed fluorescent intensity of gel coated paper discs without $\mathrm{H}_{2} \mathrm{O}_{2}$, and $\mathrm{b}$ is the slope of the plot. The equation of linear fit is,

$y=2601.83 x+40721.55\left(R^{2}=0.97537\right)$

So,

$\mathrm{LOD}=(3 * 605.16) / 2601.83 \mu \mathrm{M}=698 \mathrm{nM}$ 


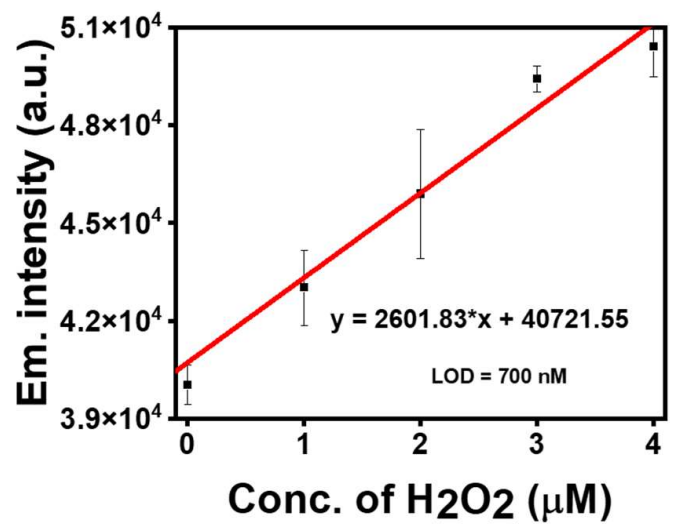

Figure S6: LOD determination plot.

\section{Calculation of LOD (using ImageJ software derived data):}

The equation of linear fit is,

$y=1.38 x+44.49\left(R^{2}=0.97808\right)$

Standard deviation of blank $(\sigma)=0.93632$

Slope of the plot $(b)=1.38$

$\mathrm{LOD}=3 \sigma / b=(3 * 0.94) / 1.38 \mu \mathrm{M}=2 \mu \mathrm{M}$

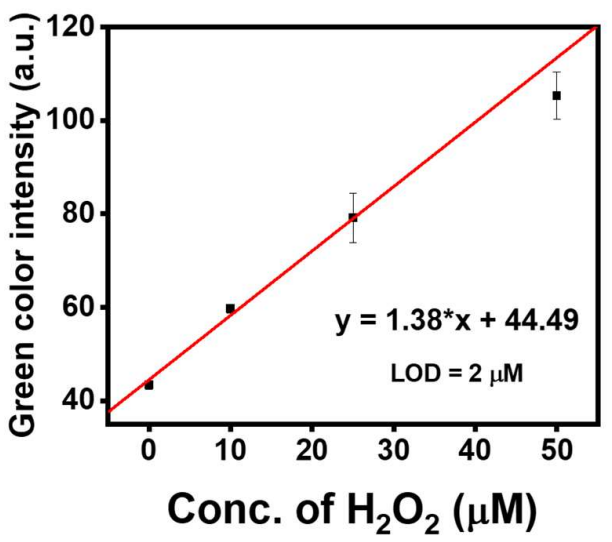

Figure S7: LOD determination plot. 
10. Table S2: Compositions of hand sanitizers.

\begin{tabular}{|c|c|c|c|c|}
\hline $\begin{array}{c}\text { Sanitizer } \\
\text { brand }\end{array}$ & $\begin{array}{c}\text { Composition as per the } \\
\text { label }\end{array}$ & $\begin{array}{c}\text { Manufacturing } \\
\text { date }\end{array}$ & Expiry date & $\begin{array}{l}\text { Date of } \\
\text { analysis }\end{array}$ \\
\hline $\mathbf{A}$ & $\begin{array}{l}\text { Denatured ethanol IP: } 80 \% \\
(\mathrm{v} / \mathrm{v}) \text {, Hydrogen peroxide: } \\
0.125 \%(\mathrm{v} / \mathrm{v}) \text {, Glycerol IP: } \\
1.45 \% \% \text {, Fragrance, } \\
\text { Excipients }\end{array}$ & October 2020 & September 2022 & $\begin{array}{l}28^{\text {th }} \text { January } \\
2021\end{array}$ \\
\hline B & $\begin{array}{l}\text { Isopropyl alcohol IP: } 75 \% \\
\text { (v/v), Hydrogen peroxide: } \\
0.125 \%(\mathrm{v} / \mathrm{v}) \text {, Glycerol IP: } \\
1.45 \%(\mathrm{v} / \mathrm{v})\end{array}$ & April 2020 & March 2023 & $\begin{array}{l}28^{\text {th }} \text { January } \\
2021\end{array}$ \\
\hline $\mathbf{C}$ & $\begin{array}{l}\text { Ethyl alcohol IP: } 96-83.33 \\
\% \quad(\mathrm{v} / \mathrm{v}) \text { equivalent to } \\
\text { absolute alcohol } 80 \%(\mathrm{v} / \mathrm{v}), \\
\text { Hydrogen peroxide: } 0.125 \\
\%(\mathrm{v} / \mathrm{v}) \text {, Glycerol IP: } 1.45 \\
\%(\mathrm{v} / \mathrm{v}) \text {, DM water Q.S., } \\
\text { Excipient Q.S. }\end{array}$ & June 2020 & May 2022 & $\begin{array}{l}28^{\text {th }} \text { January } \\
2021\end{array}$ \\
\hline D & $\begin{array}{l}\text { Ethyl alcohol IP: } 80 \% \\
\text { (v/v), Hydrogen peroxide: } \\
0.125 \%(\mathrm{v} / \mathrm{v}) \text {, Glycerol IP: } \\
1.45 \% \text { (v/v), Purified } \\
\text { water: Q.S. }\end{array}$ & April 2020 & March 2022 & $\begin{array}{l}28^{\text {th }} \text { January } \\
2021\end{array}$ \\
\hline $\mathbf{E}$ & $\begin{array}{l}\text { Ethanol IP: } 80 \%(\mathrm{v} / \mathrm{v}) \text {, } \\
\text { Hydrogen peroxide: } 0.125 \\
\%(\mathrm{v} / \mathrm{v}) \text {, Glycerol IP: } 1.45 \\
\% \text { (v/v), Purified water: } \\
\text { Q.S. }\end{array}$ & July 2020 & June 2023 & $\begin{array}{l}28^{\text {th }} \text { January } \\
2021\end{array}$ \\
\hline
\end{tabular}




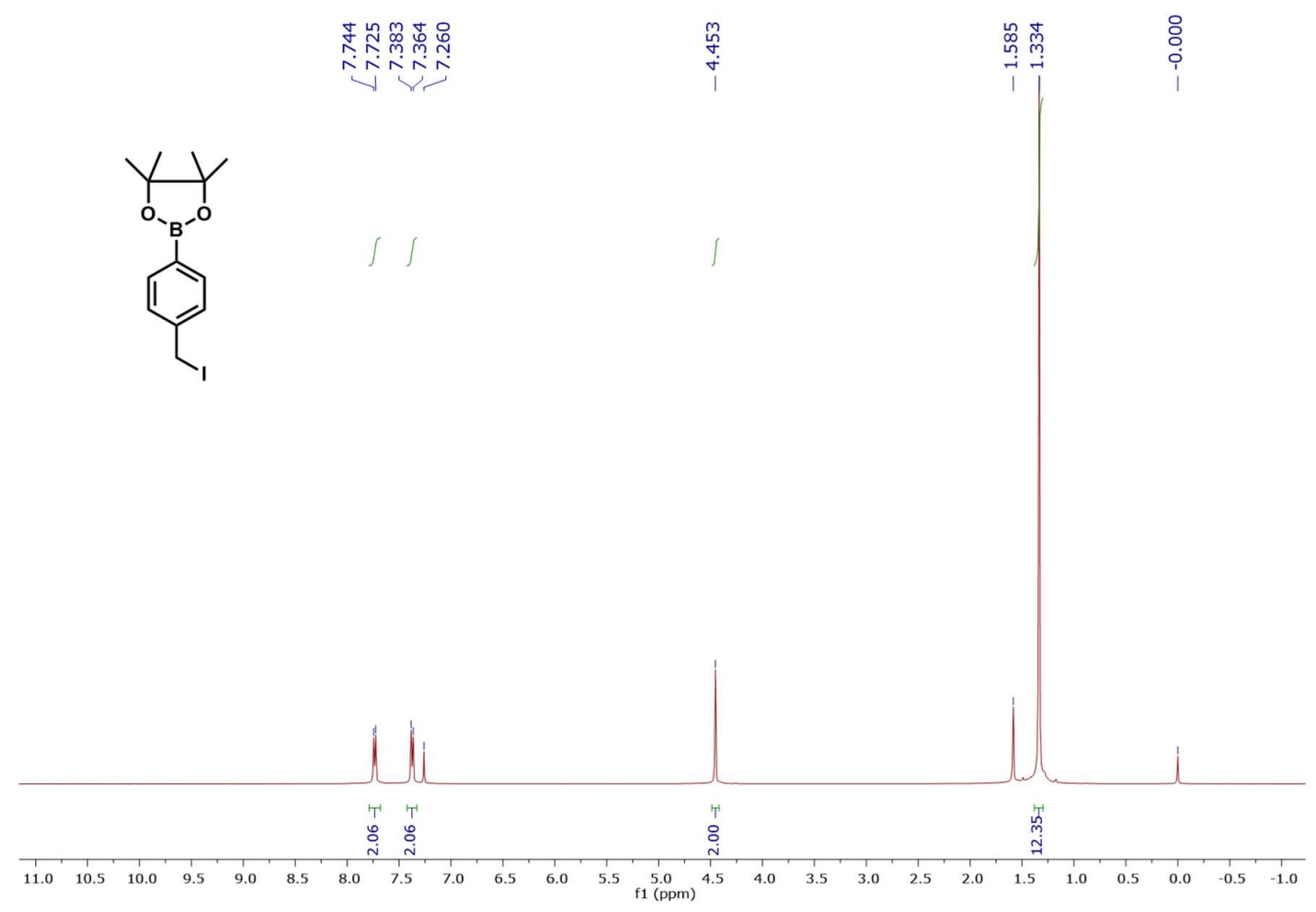

Figure S8: ${ }^{1} \mathrm{H}$ NMR spectrum of 4-(iodomethyl)phenylboronic acid pinacol ester $\left(\mathrm{CDCl}_{3}, 400 \mathrm{MHz}\right)$.

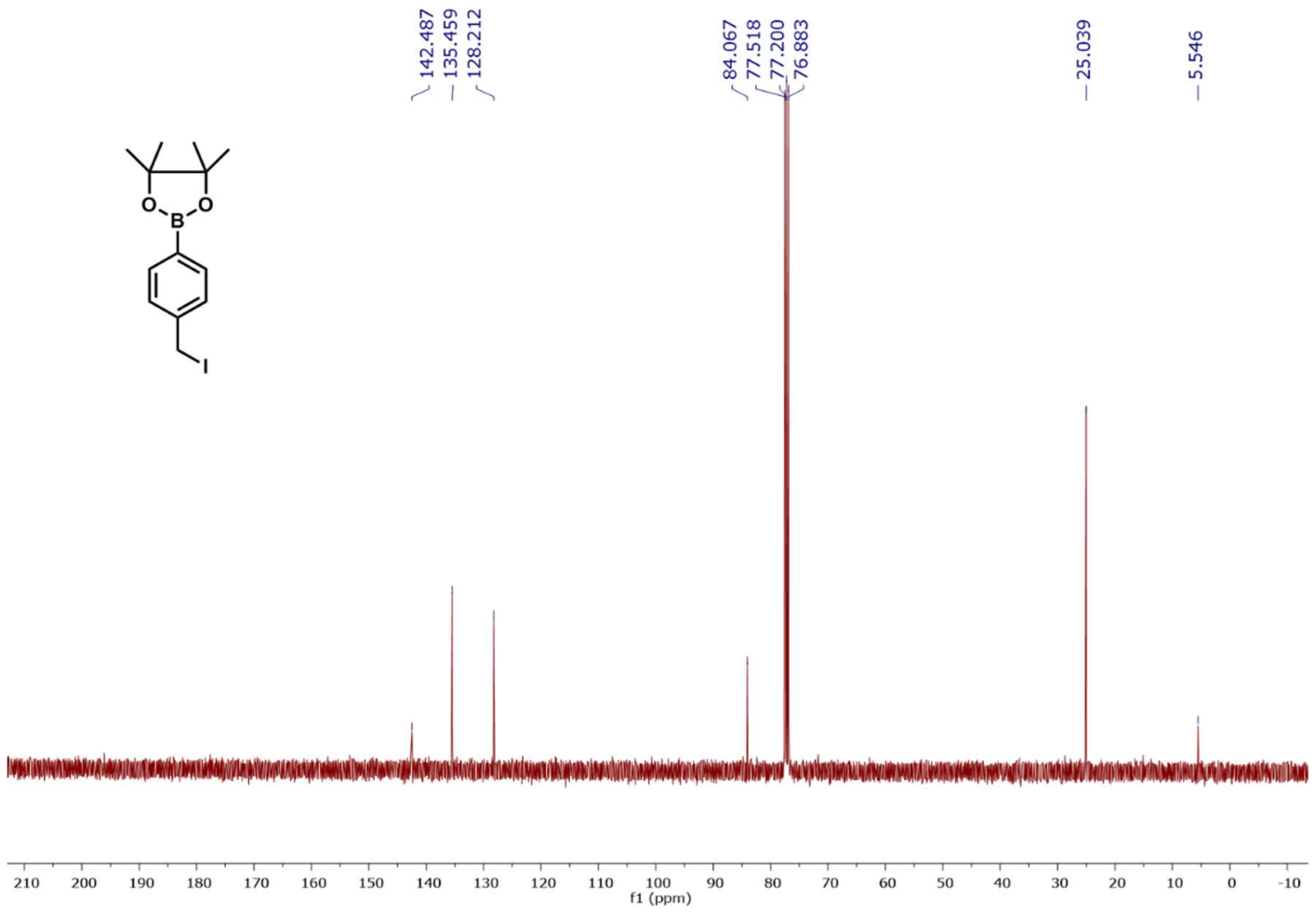

Figure S9: ${ }^{13} \mathrm{C}$ NMR spectrum of 4-(iodomethyl)phenylboronic acid pinacol ester $\left(\mathrm{CDCl}_{3}, 100 \mathrm{MHz}\right)$. 


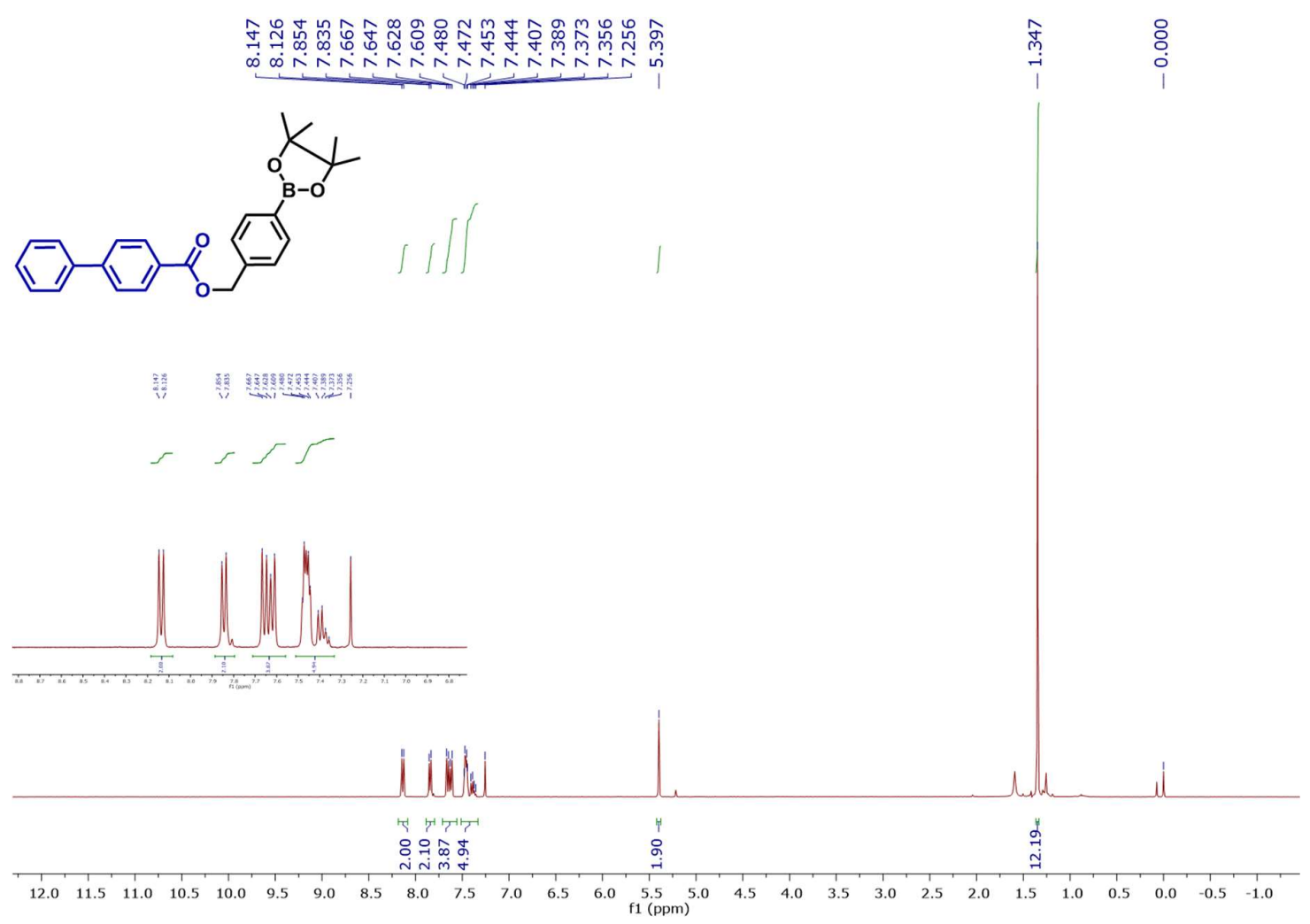

Figure S10: ${ }^{1} \mathrm{H}$ NMR spectrum of $2\left(\mathrm{CDCl}_{3}, 400 \mathrm{MHz}\right)$.
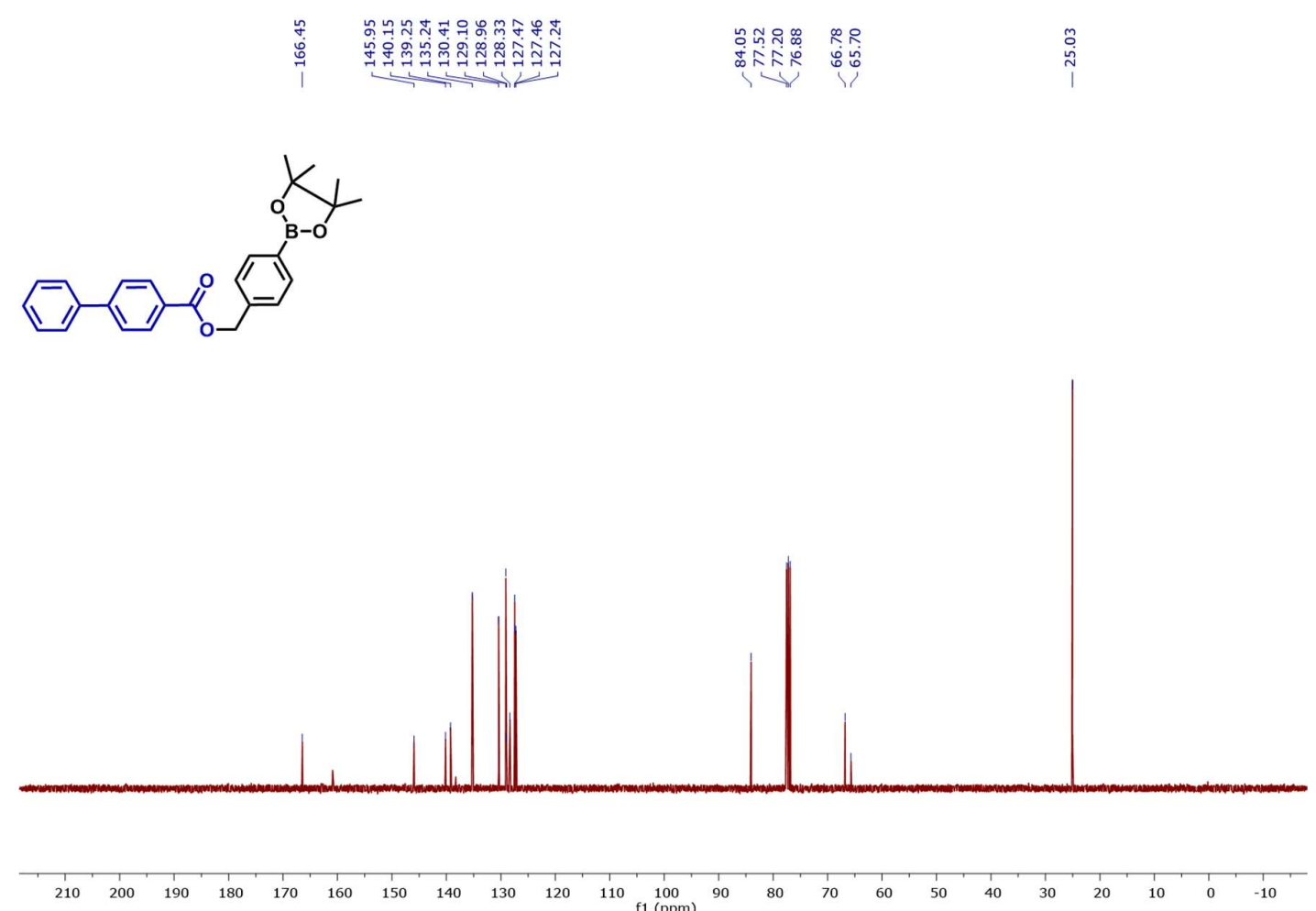

Figure S11: ${ }^{13} \mathrm{C}$ NMR spectrum of $2\left(\mathrm{CDCl}_{3}, 100 \mathrm{MHz}\right)$. 


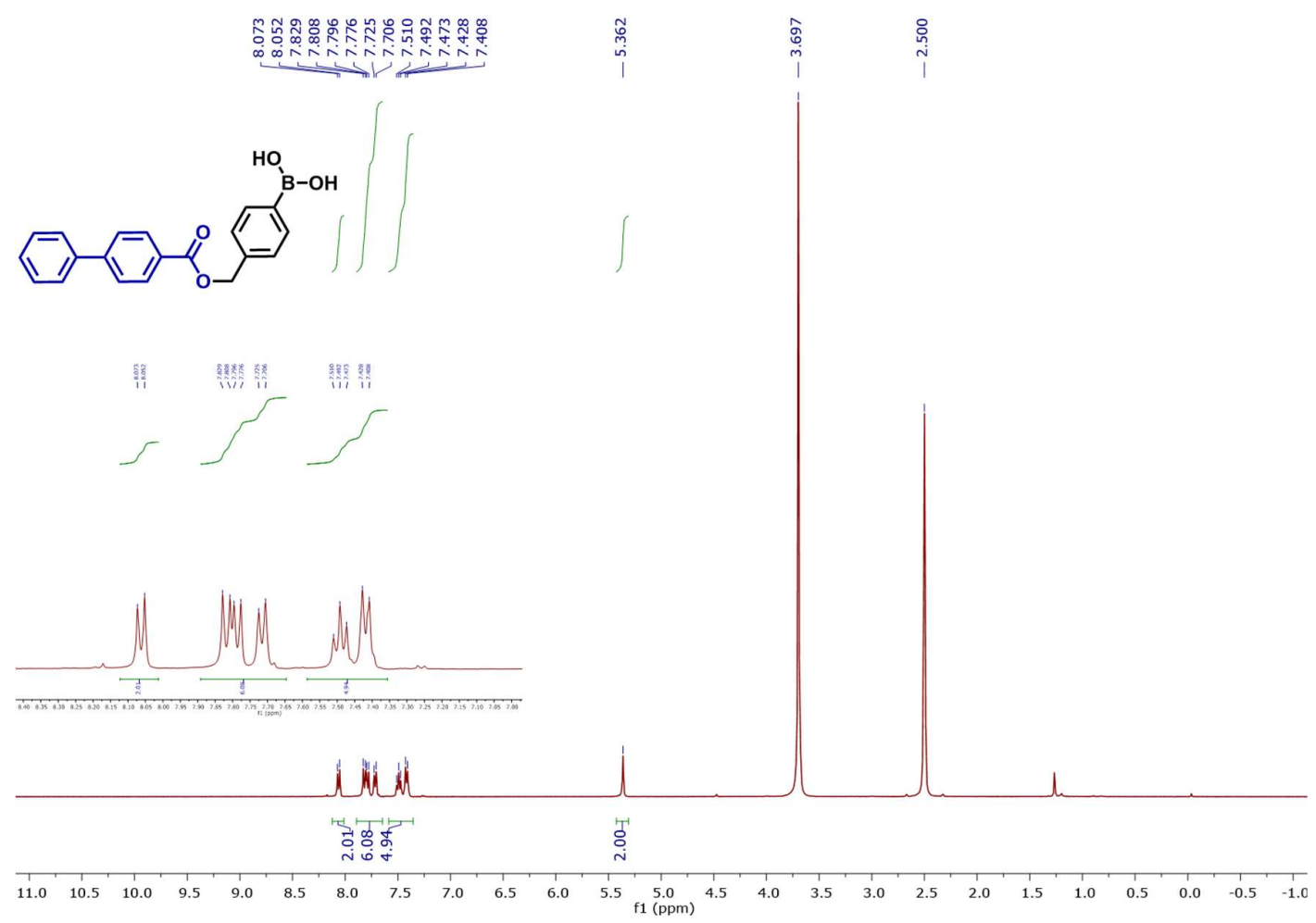

Figure S12: ${ }^{1} \mathrm{H}$ NMR spectrum of 2' (DMSO-D 6 with added $\mathrm{D}_{2} \mathrm{O}, 400 \mathrm{MHz}$ ).
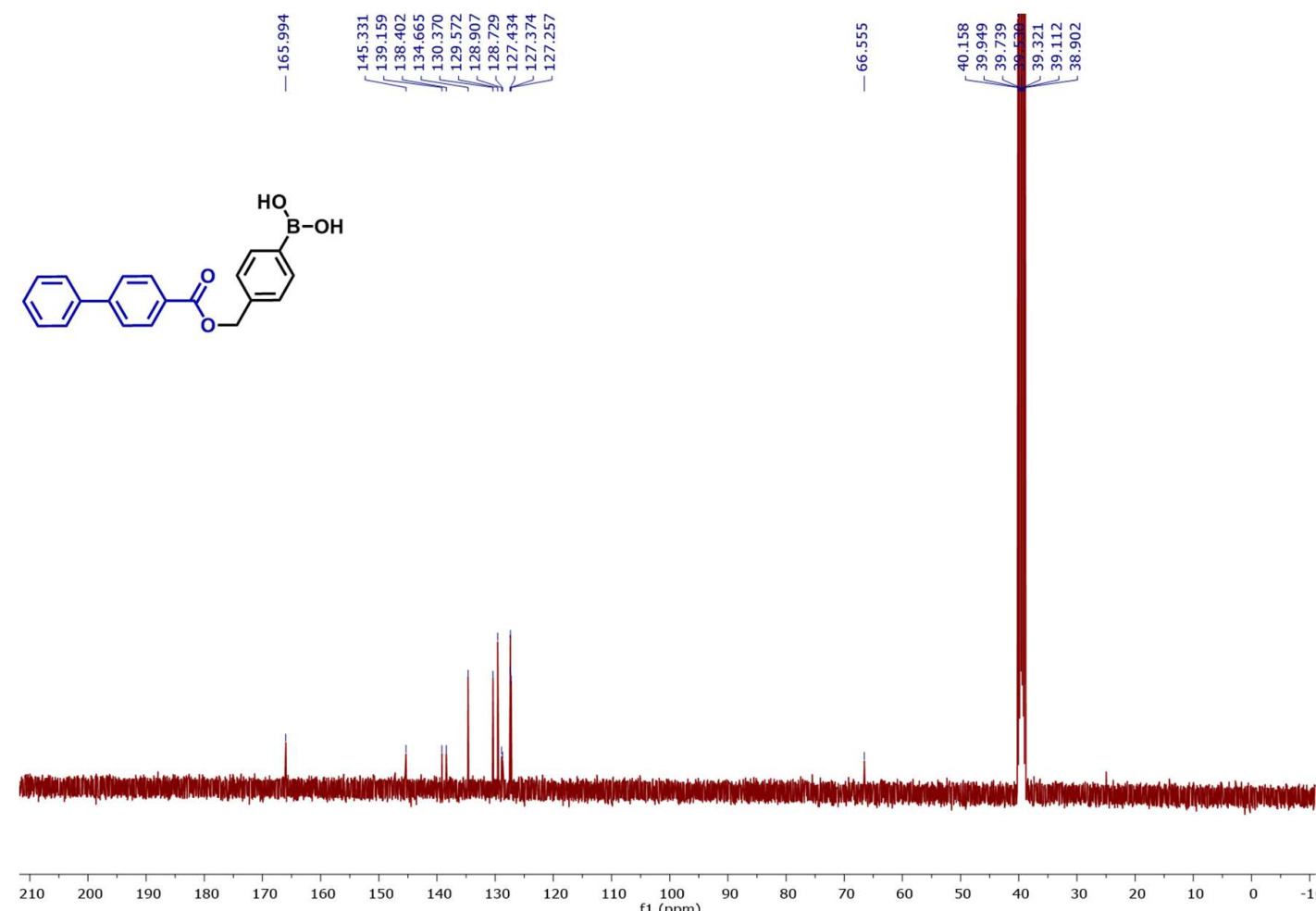

Figure S13: ${ }^{13} \mathrm{C}$ NMR spectrum of 2' (DMSO-D 6 with added $\mathrm{D}_{2} \mathrm{O}, 100 \mathrm{MHz}$ ). 